\title{
Balloon-Occluded Retrograde Transvenous Obliteration (BRTO): A Novel Method of Obliterating Gastric Varices. Report of Six Cases and Review of Literature
}

\author{
Ajit Thapa', Dinesh Koirala2", Rahul Pathak2², Dinesh Chataut', Sashi Sharma ${ }^{2}$, Brindeswari Kafle Bhandari', Anurag \\ Jha $^{2}$, Rabin Hamal' ${ }^{2}$, Rajesh Pandey'2, Arun Gyawali², Mohan Bhusal ${ }^{2}$
}

Department of Radiology and Imaging ${ }^{1}$, Department of Gastroenterology and Hepatology ${ }^{2}$

Maharajgunj Medical Campus, TUTH, Institute of Medicine, Kathmandu, Nepal

Keywords: balloon-occluded retrograde transvenous obliteration, gastric varices

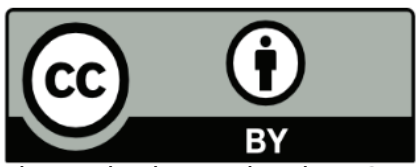

This work is licensed under a Creative Commons Attribution 4.0 Unported License.

\begin{abstract}
Portal hypertension results in various complications, gastroesophageal varices being one of them. Although less common than esophageal varices, gastric varices are difficult to obliterate and carry a higher mortality rate when bleeding occurs. They are less amenable to sclerotherapy, endoscopic variceal ligation. Balloon-Occluded Retrograde Transvenous Obliteration (BRTO) has been developed as a minimal invasive procedure to obliterate gastric varices. BRTO is an endovascular procedure where a balloon catheter is inserted into a draining vein of gastric varix, and the sclerosant can be injected into the varices through the catheter during balloon occlusion. We report six cases where BRTO was done for gastric varices obliteration.
\end{abstract}

\section{Introduction}

Gastric varices (GV) develop in approximately $20 \%$ of patients presenting with portal hypertension. ${ }^{1}$ Despite occurring at lesser frequency than esophageal varices (EV), GV bleeding is more difficult to manage endoscopically, has increased mortality and rate of rebleeding than esophageal variceal bleeding. 1 In case of gastric varices, blood flows through the gastric varices and into the systemic circulation generally through the left renal vein or the inferior vena cava. Gastric varices drain directly into large veins without intervening smaller veins, and therefore blood flow and mortality are high due to a greater blood loss when the varices rupture. ${ }^{2}$ For the same reason, endoscopic injection sclerotherapy solution if injected is flushed out immediately and exerts less sclerosing effect compared to injecting into esophageal varices.

Balloon-Occluded Retrograde Transvenous Obliteration (BRTO) is an effective and considerably safe procedure for treating gastric varices. ${ }^{3}$ BRTO involves temporary occlusion of outflow veins of the portosystemic shunt followed by endovascular injection of a sclerosant into the varix. Over the last 2 decades, BRTO has become a common modality used for the prevention and treatment of bleeding GV. Reports showing failure of BRTO are rare. We report six cases of GV in patients with cirrhosis with portal hypertension who underwent BRTO for primary and secondary prophylaxis.

\section{Description of cases}

\section{Case 1:}

43 years male with cirrhosis of liver due to alcohol presented with upper gastrointestinal (UGI) bleeding. After patient was adequately resuscitated UGI endoscopy was done which showed small esophageal varices without red color sign (RCS) which indicated that esophageal varices had not bled but nodular gastric varices (GOV2) with RCS were found along the greater curvature with RCS. This indicated that the reason for UGI bleeding was due to rupture of gastric varices. Endoscopic glue injection was planned and done accordingly. On reviewing UGI endoscopy after 1 week there was no bleeding but the varices

\footnotetext{
*Corresponding Author:

Dr Dinesh Koirala, MBBS, MD,

Department of Gastroenterology and Hepatology,

Maharajgunj Medical Campus, TUTH, Institute of Medicine,

Maharajgunj, Nepal

Email:dkoirala045@gmail.com, Phone No-+977-9849877571
} 
were not completely obliterated. Contrast enhanced CT (CECT) scan was done which showed cirrhotic liver with minimal ascites with large lienorenal shunt (LRS) with multiple small draining collaterals veins (Type B gastric varices). BRTO was planned as a method for secondary prophylaxis to prevent bleeding from gastric varices.

Right femoral vein was accessed with $8 \mathrm{~F}$ Balkin sheath. Using 5 F Cobra 1 and hydrophilic guidewire diagnostic angiographic catheter, left renal vein and subsequently LRS was cannulated. Hydrophilic guide wire was exchanged for $260 \mathrm{~cm}$ Amplatz extra stiff guidewire wire. Over the wire $16 \mathrm{~mm}$ occlusion balloon was placed in the narrowest portion of LRS ostium. Venography after balloon inflation showed opacification of gastric varices and drainage through inferior phrenic collaterals vein, which was catheterized using $2.1 \mathrm{~F}$ microcatheter and embolised using coils. The coils got displaced so it was removed with snare. Embolization was done again with gelfoam which was successful. Repeat venography showed good opacification of gastric varices and good contrast retention. $25 \mathrm{ml}$ of sclerosant i.e. mixture of lipiodol, sodium tetradecyl sulfate and air in ratio of 1:2:3 was injected into the varices through balloon catheter. Femoral sheath and catheter was secured. Plain CT after 6 hours revealed good hold up of sclerosant in the gastric varices. Balloon was deflated and removed under fluoroscopic guidance after $12 \mathrm{hrs}$. Sheath was removed and hemostasis in femoral vein puncture site was achieved by manual compression.

After observing in ward for 2 days patient was discharged on beta blocker as well. Monthly follow up was done. Repeat UGI endoscopy was done after one month which showed complete obliteration of gastric varices without worsening of esophageal varices. He had no recurrent variceal bleed till next 6 month follow up without worsening of esophageal varices, ascites and new occurrence of pleural effusion.

\section{Case 2}

60 years female with Hepatitis C related cirrhosis of liver was evaluated for the presence of gastroesophageal varices. On UGI endoscopy large tumorous isolated gastric varix (IGV 1) was found. CECT abdomen was done which showed cirrhotic liver with ascites with large LRS without draining collateral veins (Type A gastric varices). BRTO was planned and done accordingly without any significant complications. Patient complained of slight pain abdomen which got relieved conservatively. Patient was observed for few days and discharged. UGI endoscopy done after one month showed complete obliteration of the varices. No other complications were found on follow up for six months.

\section{Case 3}

60 years female with diabetes mellitus and NASH related cirrhosis with prior history of UGI bleeding 1 month back managed in some other center medically presented for evaluation. On UGI endoscopy large tumorous isolated gastric varix (IGV 1) was found. CECT abdomen was done which showed cirrhotic liver with ascites with large LRS with small draining collateral vein (Type B gastric varices). BRTO was planned and done accordingly along with gelfoam embolization to draining collateral vein without any significant periprocedural complications. UGI endoscopy done after one month showed complete obliteration of the varices. No other complications were found on follow up for six months.

\section{Case 4}

60 years female with newly diagnosed cirrhosis due to NASH cirrhosis presented with decompensation of liver disease with ascites. On UGI endoscopy three columns of esophageal varices (2 large and 1 small) with large tumorous gastric varix at fundus (GOV2) without RCS was found. BRTO was planned to obliterate the gastric varix first. BRTO was done accordingly but patient developed retroperitoneal bleeding due to rupture of LRS. This complication was managed conservatively. UGI endoscopy done after one month showed complete obliteration of the varices but it showed three columns of large esophageal varices compared to two large and one small esophageal varices preprocedure. Patient also complained of increase in abdominal distention. Two sessions of endoscopic variceal ligation was done for esophageal varices. Diuretics was optimized along with strict salt restriction. On follow up esophageal varices ere obliterated along with decrease in ascites. No other complications were found on follow up for six months.

\section{Case 5}

75 years male with hypertension, diabetes mellitus and NASH related cirrhosis was evaluated for the presence of gastroesophageal varices. On UGI endoscopy three columns of small esophageal varices with large tumorous gastric varices without RCS was found. CECT abdomen was done which showed cirrhotic liver with ascites with large LRS with small draining collateral vein (Type B gastric varices). BRTO was planned to obliterate the gastric varices. BRTO was done along with gelfoam embolization to draining collateral vein accordingly without any significant periprocedural complications. UGI endoscopy done after one month showed complete obliteration of the varices but it showed three columns of large esophageal varices compared small esophageal varices preprocedure. Three sessions of endoscopic variceal ligation was done for esophageal varices. On follow up esophageal varices were obliterated. No other complications were found on follow up for six months.

\section{Case 6}

50 years old male with alcohol related chronic liver disease presented with history of hematemesis. After stabilization UGI endoscopy showed

abundant isolated gastric varices (IGV1) with no active bleeding and was not amenable for endoscopic intervention. CECT abdomen showed features of cirrhosis with minimal perihepatic free fluid, large lienorenal shunt with isolated gastric varices indenting into lumen without draining collateral veins (Type $A$ gastric varices). BRTO was planned and done accordingly without any significant periprocedural complications. UGI endoscopy done after one month showed complete obliteration of the varices. Patient complained of increased abdominal distention which was found to be due to increase in ascites. This was controlled by dietary modification and optimizing diuretics. No other complications were found on follow up for six months. 


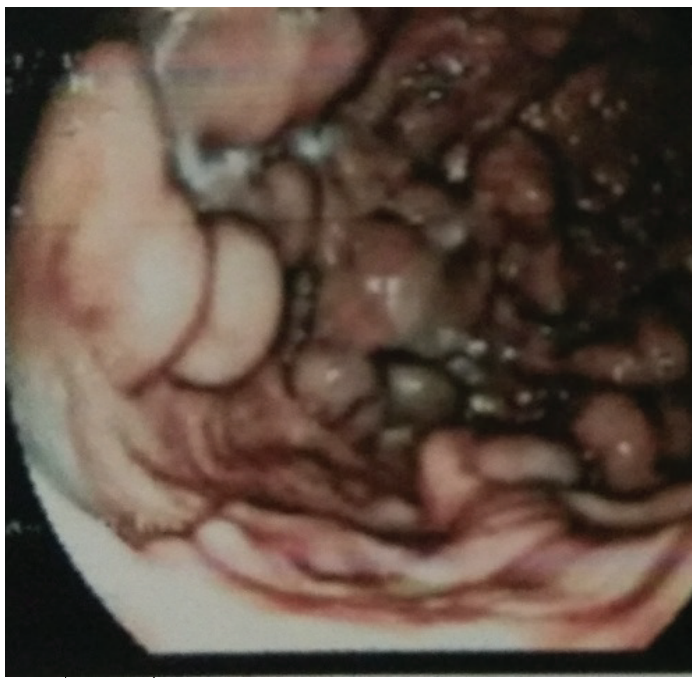

Figure 1: Endoscopic image showing gastric varices

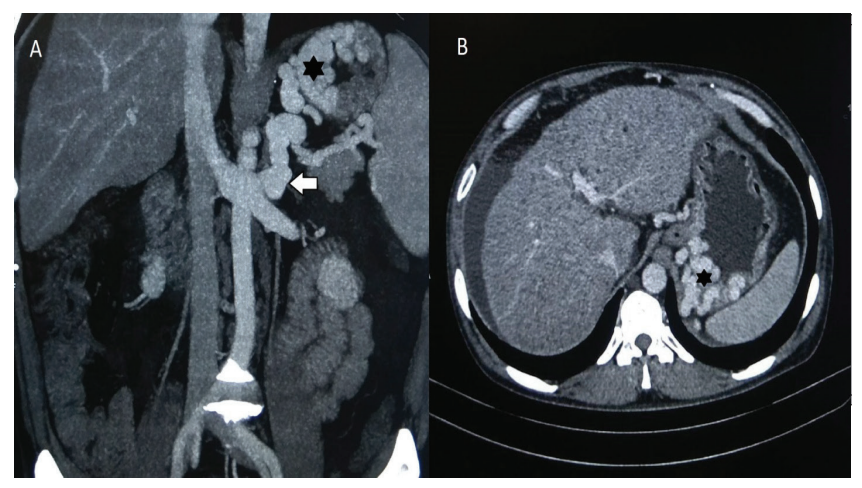

Figure 2: A. Coronal reformatted CT portogram showing lienorenal shunt (white solid arrow) and fundal gastric varices (black asterisk) B. Axial CT portogram showing fundal gastric varices indenting in gastric lumen (black asterisk)

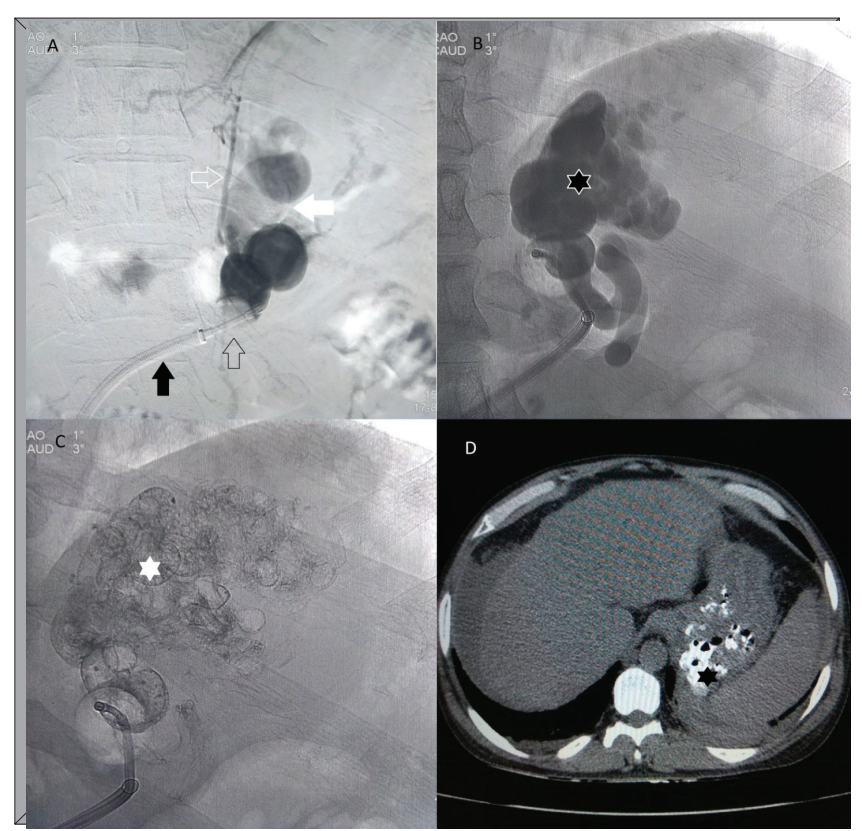

Figure 3: A. Balloon occlusion venography using $8 \mathrm{~F}$ Balkin sheath (black solid arrow) and occlusion balloon (black open arrow) showing fundal gastric varices (white solid arrow) and inferior phrenic collateral (white open arrow) B. Balloon occlusion venography after embolization of inferior phrenic collateral showing good hold up of contrast in fundal gastric varices (black asterisk) C. Fluoroscopic image showing hold up of sclerosant in gastric varices (white asterisk) D. Plain CT scan showing retention of sclerosant i.e. lipiodol, Sodium tetradecyl sulfate and air mixture in fundal gastric varices ( black asterisk)

\section{Discussion:}

Gastric varices (GV) are submucosal venous saccules in the wall of the stomach, which develop in about $20 \%$ of patients with portal hypertension. ${ }^{1}$ Gastric varices can be classified according to various systems. According to Sarin et $\mathrm{al}^{1} \mathrm{GV}$ can be classified as either gastroesophageal varices (GOV) or isolated GV (IGV). GOV are further subdivided into two types: GOV1 (varices continuous with esophageal varices, extending down to the cardia, or lesser curvature), and GOV2 (varices extending from the esophagus toward the fundus). IGV may be found in the fundus (IGV1), or may be located elsewhere in the stomach (IGV2) such as the antrum, corpus, or around the pylorus. ${ }^{1}$ Among these, GOV1 account for most GV (75\%), however, the incidence of bleeding is significantly higher for IGV1 (78\% for IGV1 vs. 55\% for GOV2, and 10\% for GOV1 and IGV2). ${ }^{1}$ The cumulative risk for bleeding from gastric fundal varices is $16 \%, 36 \%$, and $44 \%$ at 1,3 , and 5 years, respectively. ${ }^{4}$

Gastric varices can be classified into three types 5 according to the pattern of their afferent veins. Type 1 gastric varices are supplied by a single afferent gastric vein; type 2 , by multiple afferent gastric veins; and type 3 , by single or multiple gastric veins with coexistent gastric veins that are directly contiguous with the shunt but do not contribute to the varices.

Furthermore, gastric varices can be classified into four types 6 according to the pattern of their draining veins. Type A gastric varices are contiguous with a single shunt alone, type $B$ are contiguous with a single shunt and collateral veins, type $C$ are contiguous with both the gastrorenal and gastrocaval shunts, and type $D$ are not contiguous with a catheterizable shunt. Technically, type A and type B gastric varices can be subjected to BRTO with more success compared to type $C$ and type $D$.

Compared to esophageal varices, GV bleeding occurs less frequently but is associated with a poorer prognosis. GV bleeding results in greater hemorrhage and transfusion requirements, as well as increased risk of rebleeding and higher mortality rate. Mortality associated with GV hemorrhage is higher (45-55\%) as compared to mortality rate associated with EV hemorrhage (6-15\%). Endoscopic therapy for EV has high rate of technical success (87-100\%) and a lower incidence of major complications. Ruptured GV have a high rate of rebleeding post-therapy (7-65\%). ${ }^{4}$

Majority of GV are associated with a spontaneous left-sided portosystemic shunt, which include gastrorenal, direct gastrocaval, and gastrocaval shunts via the inferior phrenic vein. ${ }^{2}$ The formation of these shunts results in relieving of portal hypertension. Among these shunts, gastrorenal shunts are the most common $(80 \%$ $85 \%)^{2}$ These shunts create an outflow from the GV to the left renal vein forming a component of the portosystemic system. The gastric variceal system, which includes the varices and gastrorenal shunt, can vary in complexity, tortuosity, size, and blood flow. A thorough understanding of the anatomy and hemodynamics of the gastric variceal system should be assessed by preprocedural imaging studies using CT before proceeding for management decisions.

Management options for acute gastric variceal bleed are 
cyanoacrylate glue injection, transjugular intrahepatic portosystemic shunt (TIPSS) and surgery. Endoscopic variceal ligation (EVL) is not preferably used for GV because rebleeding rate of EVL is significantly higher than that of endoscopic obliteration using cyanoacrylate (54\% vs. $31 \%$ ). ${ }^{5}$ TIPSS may be used as a primary treatment or as a rescue therapy if variceal bleed is not controlled by cyanoacrylate glue. The success rate of TIPSS for controlling gastric variceal bleeding is $90-96 \%$ although rates of rebleeding ranged from $20 \%$ to $30 \% .^{7}$ After glue injection therapy for GV, overlying mucosa is eroded along with extrusion of glue cast gradually. Early extrusion, within 1 week, is seen in $12.1 \%$ and is associated with early rebleeding in half of these patients $(6.2 \%)$ with late rebleeding, 2-18 months after injection in only $8.1 \% .^{5}$ There is no ample data regarding comparison of endoscopic, interventional radiologic, and surgical treatment modalities for the management of bleeding GV. This has led to different consensus among different practitioners regarding the common and optimal management strategy for this condition.

A novel method of obliterating the GV called BRTO without the inherent risks of EVL and glue therapy has emerged after its origin in Japan by Kanagawa et al. BRTO involves occlusion of the portosystemic outflow veins with a balloon catheter, followed by injection of a sclerosing agent into the varix. Mostly the venous access site commonly used are common femoral vein or internal jugular vein. After injecting sclerosant agent the occlusion balloon is kept in situ for hours to ensure that there is sufficient dwelling of the sclerosing material within the varix and to minimize complications due to reflux of the sclerosant into the systemic or portal vessels. The sclerosant results in thrombosis of the GV and draining portosystemic shunt.

Two major clinical indications for BRTO include: (1) impending, prior, or active gastric variceal bleeding, and (2) GV with hepatic encephalopathy refractory to medical management.

Relative contraindications of BRTO include: (1) severe uncorrected coagulopathy, (2) splenic vein thrombosis, (3) ascites, and (4) uncontrolled esophageal variceal bleeding. For uncontrolled esophageal variceal bleeding along with GV, BRTO can be done if it is combined with TIPSS. The most important contraindication for undergoing BRTO is chronic portal vein thrombosis as the gastrorenal shunt could be the only splanchnic outflow tract. Thus, its obliteration with BRTO poses the greatest risk for adverse consequences due to splenic engorgement, thrombosis, and venous mesenteric ischemia. If a GV lacks a main draining vein it is not suitable to perform BRTO, as they cannot be catheterized. Moreover, GV having a direct connection with the coronary vein and esophageal varices are also difficult to treat using BRTO. Reviewing these contraindication it is imperative to have good knowledge about patient's splanchnic circulation using imaging technique before progressing to the procedure.

The other endovascular radiologic management of bleeding GV includes TIPSS, which results in decompression of the portal circulation. BRTO differs from TIPSS in obliterating the varix rather than decompressing the portal circulation. Balloon-occluded antegrade transvenous obliteration (BATO) is an alternate approach that occludes the inflow to the varices from the portal system and can be performed by direct transhepatic puncture of the portal veins or via access through a TIPSS shunt.

Compared to TIPSS, BRTO has an important advantage. BRTO improves hepatic blood flow and liver function, thereby improving hepatic encephalopathy whereas TIPSS has been found to increase the incidence of hepatic encephalopathy owing to creation of portosystemic shunt.8 Furthermore, the cumulative gastric variceal bleeding rate at 1 year was $20 \%$ in patients who underwent TIPS compared with $2 \%$ in patients who underwent
BRTO. $^{8}$

But BRTO also has its fallacies. BRTO increases the risk of new-onset or worsening esophageal variceal bleeding by closing the portal outflow shunt and thus altering local hemodynamics and collateral flow.7 Thus patients who have undergone BRTO should be closely monitored for detection and management of esophageal varices. 9 Factors predicting the worsening of esophageal varices after BRTO are presence of esophageal varices, higher Child-Pugh class, and higher resistance index assessed by endoscopic color Doppler ultrasonography before BRTO.10 Worsening of esophageal varices was seen in $27 \%$ of patients at 1 year and $58 \%$ at 3 years. ${ }^{9}$ Also there is a risk of increasing ascites and pleural effusion due to increased portal pressure. Some patients might even require post-BRTO TIPSS for worsening ascites and pleural effusion if refractory to lifestyle and medical management. Common complications postBRTO include fever, epigastric, chest and/or back pain, transient systemic hypertension, and hemoglobinuria. Most of these complication resolve conservatively. Recent systematic review of 24 studies demonstrate, BRTO has excellent clinical and technical success rates (79\%-100\%) also being efficacious and relatively safe procedure for treating GV.10 Furthermore, in comparison to endoscopic-guided sclerotherapy or cyanoacrylate injection as primary treatment for GV, BRTO had a lower rebleed rate. A meta-analysis comparing BRTO to TIPS in patients with GV found these interventions to be similar in terms of technical success rate, hemostasis rate, and incidence of postoperative complications, but BRTO has an advantage with a lower incidence of rebleeding and encephalopathy. ${ }^{9}$ Rebleed rates after a successful BRTO, EIS, $\mathrm{EVL}$, and glue therapy for GV range from $0 \%-10 \%, 55 \%, 54 \%$ and $31 \%$ respectively. ${ }^{9,8,11}$ Furthermore, endoscopic-guided injection of cyanoacrylate into the varices may induce multiple organ embolisms, such as cerebral infarction and pulmonary embolisms, which are caused by leakage of the sclerosant into the systemic circulation. ${ }^{12}$ By contrast, BRTO is a minimally invasive treatment of gastric varices that is associated with a high success rate and a low recurrence rate. ${ }^{9}$

Therefore, BRTO can be considered as much more effective and with less complications as compared to surgery, TIPSS, or endoscopic treatment for critical patients.

\section{Conclusion:}

BRTO is a minimally invasive procedure for the treatment of gastric varices with high success rate, minimal complications and less need for re-intervention. With technical improvement in our country this procedure could provide a safe and effective option for treating gastric varices.

\section{Conflict of interest:}

The authors have none to declare.

\section{References}

1. Sarin SK, Lahoti D, Saxena SP, Murthy NS, Makwana UK. Prevalence, classification and natural history of gastric varices: A long-term follow-up study in 568 portal hypertension patients. Hepatology. 1992;16(6):1343-9.

2. Saad WEA. Vascular anatomy and the morphologic and hemodynamic classifications of gastric varices and spontaneous portosystemic shunts relevant to the brto procedure. Tech Vasc Interv Radiol. 2013 Jun;16(2):60-100.

3. Kanagawa H, Mima S, Kouyama H, Gotoh K, Uchida T, Okuda K. Treatment of gastric fundal varices by balloon-occluded retrograde transvenous obliteration. J Gastroenterol 
Hepatol. 1996;11(1):51-8.

4. Lee $\mathrm{CH}$, Lee $\mathrm{JH}$, Choi YS, Paik SW, Sinn DH, Lee CY, et al. Natural history of gastric varices and risk factors for bleeding. Korean J Hepatol. 2008;14(3):331-41.

5. Wani ZA, Bhat RA, Bhadoria AS, Maiwall R, Choudhury A. Gastric varices: Classification, endoscopic and ultrasonographic management. Vol. 20, Journal of Research in Medical Sciences. Isfahan University of Medical Sciences(IUMS); 2015. p. 1200-7.

6. Kiyosue $\mathrm{H}$, Mori $\mathrm{H}$, Matsumoto $\mathrm{S}$, Yamada $\mathrm{Y}$, Hori $\mathrm{Y}$, Okino Y. Transcatheter Obliteration of Gastric Varices: Part 1. Anatomic Classification. Radiographics. 2003;23(4):911-20.

7. Ryan BM, Stockbrugger RW, Ryan JM. A Pathophysiologic, Gastroenterologic, and Radiologic Approach to the Management of Gastric Varices. Gastroenterology. 2004;126(4):1175-89.

8. Ryan BM, Stockbrugger RW, Ryan JM, Tripathi D, Therapondos G, Hayes PC, et al. Tips for gastric varices [7] (multiple letters). Vol. 52, Gut. BMJ Publishing Group; 2003. p. 772.

9. Park JK, Saab S, Kee ST, Busuttil RW, Kim HJ, Durazo F, et al. Balloon-Occluded Retrograde Transvenous Obliteration (BRTO) for Treatment of Gastric Varices: Review and MetaAnalysis. Vol. 60, Digestive Diseases and Sciences. Springer New York LLC; 2015. p. 1543-53.
10. Prajapati R, Ranjan P, Gupta A, Yadav AK. Balloon-Occluded Retrograde Transvenous Obliteration (BRTO): A Novel Method of Control of Bleeding from Post-Glue Ulcer over Gastric Varices. Report of Two Cases and Review of Literature. J Clin Exp Hepatol [Internet]. 2016;6(4):326-30. Available from: http://dx.doi.org/10.1016/j.jceh.2016.08.005

11. Goral, Yilmaz. Current Approaches to the Treatment of Gastric Varices: Glue, Coil Application, TIPS, and BRTO. Medicina (B Aires) [Internet]. 2019 Jul 3 [cited 2020 May 28];55(7):335. Available from: https://www.mdpi.com/1010$660 \times / 55 / 7 / 335$

12. Al-Hillawi L, Wong T, Tritto G, Berry PA. Pitfalls in histoacryl glue injection therapy for oesophageal, gastric and ectopic varices: A review. World J Gastrointest Surg. 2016;8(11):729. 